\title{
Measurement and simulation of Lamb waves in adhesive-bonded multilayer systems
}

\author{
Henning Zeipert ${ }^{1}$, Leander Claes $^{1}$, Sarah Johannesmann ${ }^{1}$, Manuel Webersen ${ }^{1}$ \\ Yevgeniya Lugovtsova ${ }^{2}$, Jens Prager ${ }^{2}$, Bernd Henning ${ }^{1}$ \\ ${ }^{1}$ Measurement Engineering Group, Paderborn University, \\ Warburger Straße 100, 33098 Paderborn, Germany \\ ${ }^{2}$ Div. Acoustic and Electromagnetic Methods, Bundesanstalt für Materialforschung und -prüfung, \\ Unter der Eichen 87, 12205 Berlin, Germany \\ zeipert@emt.uni-paderborn.de
}

\begin{abstract}
Summary:
Lamb waves are a common tool in the field of non-destructive testing and are widely used for material characterisation. Further, the increasing computational capability of modern systems enables the simulation of complex and detailed material models. This work demonstrates the possibility of simulating an adhesive-bonded multilayer system by characterising each layer individually, and introduces an approach for determining the dispersive behaviour of acoustic waves in a multilayer system via real measurements.
\end{abstract}

Keywords: Adhesive bonds, Lamb waves, material parameter, non-destructive testing, ultrasound

\section{Motivation}

Adhesive bonding of different materials is a common method in modern technologies. To ensure those bonds meet the expectations, it is necessary to investigate their quality. A possible approach is the analysis of mode repulsion points of Lamb waves as presented by Lugovtsova et al. [1]. Methods like these rely on both precise measurements and simulations of the respective systems. The investigation presented in this contribution considers a two-layer system of an aluminium plate $(3 \mathrm{~mm})$ and a polycarbonate plate $(4 \mathrm{~mm})$. After a characterisation of each individual plate, the two samples are bonded using an epoxy adhesive and investigated as a multilayer system.

\section{Experimental method}

The measurements for this investigation are conducted with the setup developed by Claes et al. [2]. The setup uses a short laser pulse, which, due to the photoacoustic effect, generates broadband mechanical waves inside a plateshaped specimen, which are detected using a piezoelectric transducer. Optical components, which focus the laser beam as a thin line onto the specimen's surface, are mounted on a linear actuator. This enables a variation of the point of excitation on the plate sample, allowing for the acquisition of two-dimensional measurement data in temporal and spatial domain. The application of a two-dimensional Fourier transform yields information in frequency and wavenumber domain. A possible depiction of this data is called "dispersion map" and visualizes the detected lamb modes as ridges (Fig. 1).

\section{Material characterisation}

Dispersion diagrams for this investigation are calculated via the SAFE method [3], assuming a plane-strain isotropic model for the individual layers. To determine the material parameters of the layer specimens, an inverse procedure is used [4]. In an inverse procedure, the sound velocities of the material are optimised to match the dispersion curves to the ridges of the dispersion map (Fig. 1).

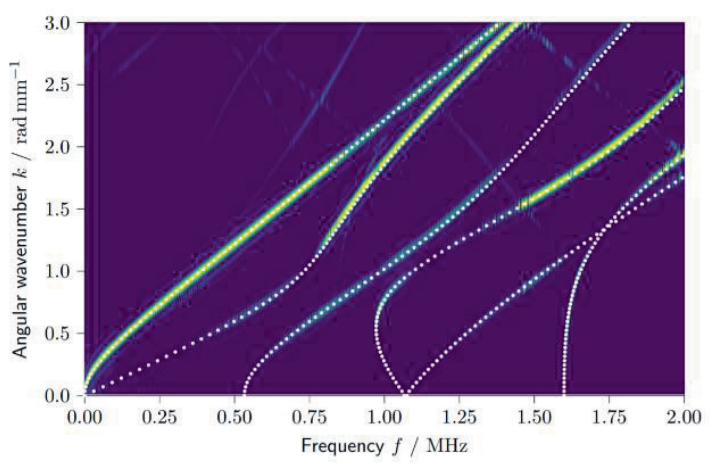

Fig. 1. Dispersion curves (white dots) based on optimised transversal and longitudinal wave velocities match the measurement based dispersion map (image) of the aluminium ( $3 \mathrm{~mm}$ ) plate.

\section{Multilayer system}

The dispersive propagation of Lamb waves inside the aluminium-polycarbonate-system is simulated with a SAFE model [3], which is built 
by stacking the two models of the layer specimens using the individually determined material parameters. The adhesive layer is neglected, thus assuming an ideal coupling of the layers. The Lamb wave excitation for the measurements are performed on the polycarbonate layer while the detecting transducer is placed on the aluminium layer. For the sake of improved visibility, Fig. 2 displays only a part of the resulting dispersion map of such a measurement, overlaid with the simulated dispersion diagram. The simulation results for the multilayer specimen are in general in good agreement with the measurement data, despite the simplifying assumption of the adhesive layer.

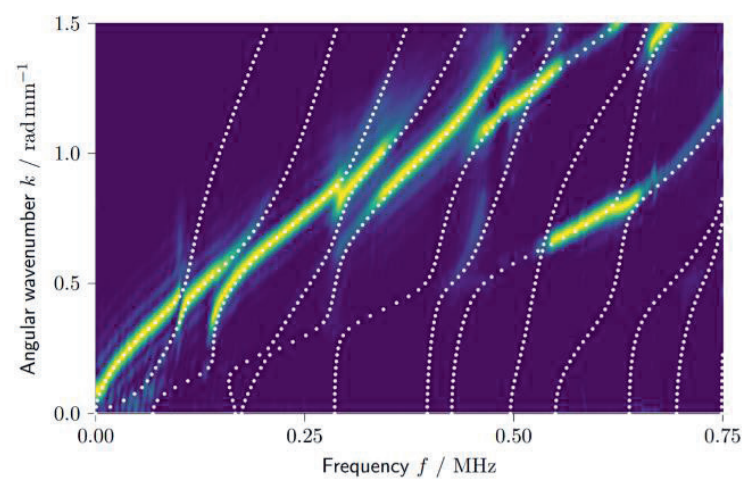

Fig. 2. Comparison of simulation (white dots) and measurement (image) of the aluminium-polycarbonate-system. The ridges in the dispersion map (image) and the simulated dispersion curves are in good agreement of one another.

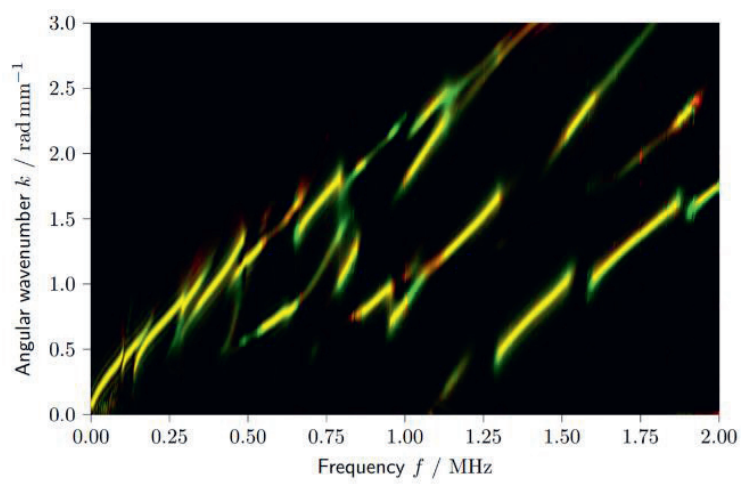

Fig. 3. Color-coded comparison of measurement results of the same specimen after $48 \mathrm{~h}$ (red) and $120 \mathrm{~h}$ (green) of adhesive curing. Yellow regions indicate matching data in both results while red/green regions are predominantly contributed by one of the results.

\section{Adhesive curing}

Since it is possible to investigate the Lamb wave propagation of multilayer systems, it will further be determined whether one can recognise a difference in the material behaviour in different stages of adhesive curing. Therefore, two different measurements of the specimen are performed. The first one about $48 \mathrm{~h}$ after application of the adhesive and a second one after about
$120 \mathrm{~h}$ after bonding. To compare those two measurements, their resulting dispersion maps are overlaid in Fig. 3 using a color-coded depection. In this, the two dispersion maps are each assigned to one RGB-channel (48 h red, $120 \mathrm{~h}$ green) and the third channel (blue) is set to zero. Due to this coding, the depiction displays black values where the two measurement are identically zero and yellow values where both feature intense signal data. For the most part the displayed measurements are identical and both feature the same modes. Further, the $120 \mathrm{~h}$ cured system provides signal data for those modes over a slightly larger range. Therefore, most of the yellow ridges start and end with a green extension, indicating an improved coupling of both plates and lower absorption.

\section{Conclusion}

The investigation at hand shows that it is possible to get detailed measurements of ultrasonic waves in adhesively bonded multilayer systems using non-destructive testing methods with laser-induced Lamb waves. Further, is it possible to simulate the dispersive behaviour of those waves if the material properties of the included layers are known in advance. Additionally, it is possible to use this measurement setup to investigate changes in the material behaviour during the curing of the adhesive bonding. For further investigations, the authors aim to derive test methods for adhesive bonding strength from the observed changes in the dispersive properties.

\section{Acknowledgements}

The authors would like to thank the German Research Foundation (DFG) for financial support of the research project 449607253.

\section{References}

[1] Y. Lugovtsova, S. Johannesmann, B. Henning, J. Prager, Analysis of Lamb wave mode repulsion and its implications to the characterisation of adhesive bonding strength, International Congress on Ultrasonics (2019); doi: 10.1121/2.0001074

[2] L. Claes, T. Meyer, F. Bause, J. Rautenberg, B. Henning, Determination of the material properties of polymers using laser-generated broadband ultrasound, Journal of Sensors and Sensor Systems (2016), doi: 10.5194/jsss-5-187-2016

[3] I. Bartoli, A. Marzani, F. Di Lanza Scalea, E. Viola, Modeling wave propagation in damped waveguides of arbitrary cross-section, Journal of Sound and Vibration (2006), doi: 10.1016/j.jsv.2006.01.021

[4] M. Webersen, S. Johannesmann, J. Düchting, L. Claes, B. Henning, Guided ultrasonic waves for determining effective orthotropic material parameters of continuous-fiber reinforced thermoplastic plates, Ultrasonics (2018), doi: 10.1016/j.ultras.2017.10.005 